\title{
Métrologie et Pédagogie
}

\author{
Carole BEAULIEU ${ }^{1, *}$ and Marie-Aude ENGELS ${ }^{2, *}$ \\ ${ }^{1}$ LNE, Pôle des Eco-Industries, 3 rue Raoul Follereau, 86100 Poitiers, France \\ ${ }^{2}$ LNE, 1 rue Gaston Boissier, 75015 Paris, France
}

\begin{abstract}
What do metrologists and pedagogues have in common? These terms have multiple evocations that need to be clarified. Both are facilitators. The metrologist is the conductor of the measurement management system in his company. The pedagogue accompanies the change by choosing tools adapted to learners specificities. Both have the function of developing a strategy to track compliance through the measurement of deviations. The metrologist helps to demonstrate compliance of the product with the specified requirements by providing the appropriate measurement strategy. The pedagogue develops a learning strategy to enable the learner to gain competence. Both activities are at the service of other entities. The metrologist is at the service of production just as the pedagogue is at the service of a content. This involves mastering the project management and benevolent communication techniques. They must be able to create a conducive environment to ease the development of collective intelligence. A training course in metrology must guarantee the performance of measurement, testing and analysis results using play tools that stimulate the creativity and assimilation of learners. The objective is to propose to metrology managers, often multifunctional, an overview of their responsibilities in order to help them (re)discover the large range of their function.
\end{abstract}

\section{Introduction}

Qu'ont en commun la métrologie et la pédagogie?

La métrologie est la science des mesures (et ses applications), la pédagogie est la science de l'éducation... à priori ce sont des disciplines qui n'ont pas grandchose en commun.

\subsection{Méconnaissance de la métrologie}

\subsubsection{La carence scolaire}

On observe dans les entreprises et, plus généralement, dans la société une méconnaissance de ce qu'est la métrologie, de ce qu'elle représente et de ce qu'elle implique. Cette inculture métrologique trouve en partie une explication dans l'absence de l'enseignement de la discipline, même dans l'enseignement supérieur. Nos sociétés ont simplement oublié d'enseigner la métrologie [1].

\subsubsection{La restriction dans les entreprises}

A cela s'ajoute la certitude dans l'entreprise que la métrologie concerne les instruments de mesure. Pour cette raison, la fonction métrologie est souvent limitée à la gestion des raccordements métrologiques. Le métrologue d'entreprise est le gestionnaire des étalonnages au lieu d'être le garant de la fiabilité des résultats de mesure. Cette restriction ne permet pas de donner à la métrologie d'entreprise toute sa dimension, c'est-à-dire qu'elle est subie au lieu de permettre la prise de décisions objectives. La gestion des équipements est elle-même gérée sans toute la cohérence nécessaire. Les raccordements métrologiques et les opérations de surveillance ne sont pas traités sous l'angle de la criticité, ce qui permettrait de déployer davantage de moyens pour les processus de mesure qui ont un impact significatif sur la qualité du produit.

\subsubsection{Une méconnaissance généralisée}

Ces constats expliquent le manque de connaissances de la société en général et des entreprises en particulier sur la métrologie.

\subsection{Complexité de la pédagogie}

\subsubsection{Eduquer, former ou enseigner?}

Eduquer, c'est former l'esprit de quelqu'un, développer ses aptitudes intellectuelles, physiques, faire acquérir des principes moraux. Former, c'est inculquer à quelqu'un les principes, les habitudes, les connaissances qui développent ses aptitudes, son goût,... Enseigner, c'est faire acquérir la connaissance ou la pratique.

La pédagogie est l'ensemble des méthodes utilisées pour éduquer les enfants et les adolescents ou une pratique éducative dans un domaine déterminé, une méthode d'enseignement.

\footnotetext{
Corresponding author: carole.beaulieu@lne.fr $\quad$ marie-aude.engels@,Ine.fr
}

C The Authors, published by EDP Sciences. This is an open access article distributed under the terms of the Creative Commons Attribution License 4.0 (http://creativecommons.org/licenses/by/4.0/). 
L'andragogie est la science et l'art d'enseigner aux adultes.

Cependant, le terme pédagogie est généralement utilisé pour évoquer l'andragogie. C'est un abus de langage que nous conserverons dans tout le présent article.

L'apprentissage est l'initiation par l'expérience à une activité. [2]

La Formation Professionnelle Continue est ouverte à toute personne, jeune ou adulte déjà engagée dans la vie adulte.

Le rappel de ces quelques définitions montre la complexité du champ de la formation professionnelle.

\subsubsection{La place du savoir}

Cependant, depuis l'origine, la préoccupation est la même. Il s'agit de donner les moyens à quelqu'un de grandir, d'évoluer. L'étymologie du terme «pédagogie» vient de deux mots grecs "paidos = enfant » et "gogia $=$ mener/conduire » ; son concept désignait l'esclave qui accompagnait les enfants à l'école. Dans toute démarche de formation, il y a toujours un changement, une évolution qui est visée par le formateur. $\mathrm{Si}$ les techniques changent, les problématiques restent les mêmes : le savoir est-il chez le maître ou chez l'élève ? La pédagogie est-elle la transmission du savoir du formateur à l'apprenant ou la création par le formateur d'un environnement qui permettra à l'élève d'accéder seul au savoir ? [3]

\subsubsection{L'apprenant au cœur du dispositif de formation}

Le champ de la formation s'appuie sur des domaines variés tels que la psychologie, la sociologie, le management, les organisations. Parmi les points de vigilance qui ont traversé le temps, 4 touchent ces différents domaines et sont particulièrement importants.

Tout d'abord, il y a 3 types de savoirs [savoir, savoirêtre, savoir-faire] et une compétence est généralement acquise lorsque les 3 savoirs sont maîtrisés. Dans le cadre d'une formation qualifiante, le formateur doit mesurer l'acquisition des trois savoirs.

Illustration 1. Les 3 types de savoirs

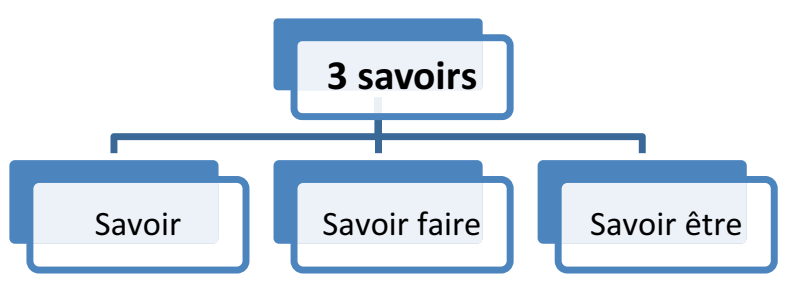

Le deuxième point de vigilance du formateur porte sur la façon de raisonner des apprenants. Tout le monde ne raisonne pas spontanément de la même façon et il est important que le formateur varie son intervention en utilisant ces différents modes de raisonnement pour permettre à chacun de comprendre et mémoriser le contenu.

Illustration 2. Les 4 modes de raisonnement

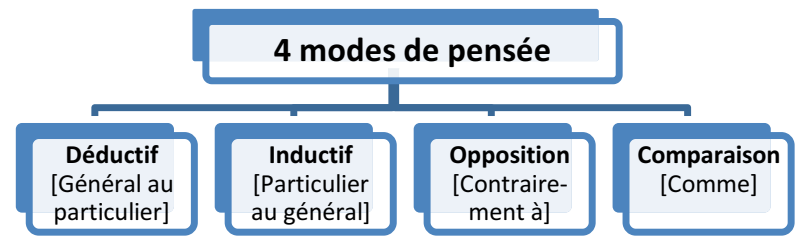

Le troisième point concerne les modes de transmission $\mathrm{du}$ savoir. Le formateur doit utiliser des modes de transmission du savoir multiples. Demander à un auditoire d'écouter, d'observer, de comparer, d'élaborer une réponse argumentée, de pratiquer par essais/erreurs alternativement permet d'évaluer les 3 savoirs.

Illustration 3. Les méthodes de transmission

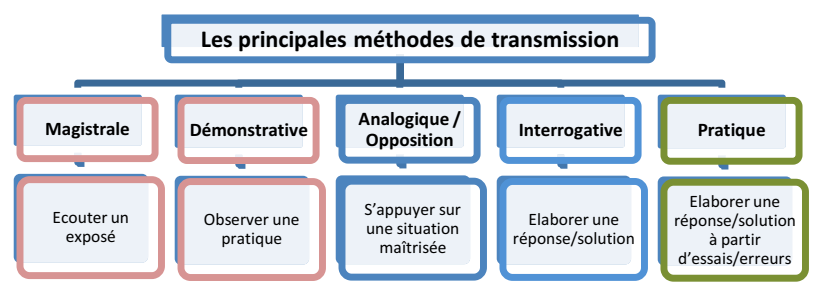

En outre, un adulte n'apprend pas tout à fait de la même façon qu'un enfant ou un adolescent, le formateur doit en être conscient. Il est nécessaire d'instaurer un climat de confiance, une dynamique qui impose un rythme soutenu et des échanges fructueux et chaleureux.

Illustration 4. Les grands principes de l'andragogie [4]

LES GRANDS PRINCIPES DE L'ANDRAGOGIE

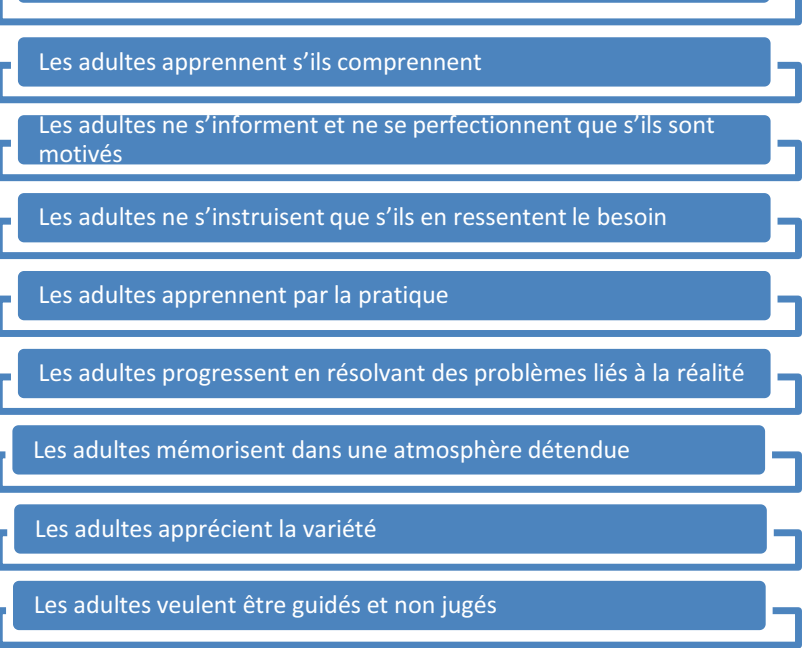

La complexité de la pédagogie vient de la nécessité d'adapter la façon de transmettre un savoir au plus près de la spécificité de chaque individu pour lui permettre d'évoluer. 


\subsection{Des similitudes}

La science des mesures (et ses applications) et la science de l'éducation ont donc une première similitude : la méconnaissance. En effet, la métrologie est un sujet globalement méconnu tout comme la complexité de la pédagogie est méconnue. Le périmètre et la finalité sont souvent flous, la vision en entreprise est erronée. La métrologie et la pédagogie sont pourtant des sciences fondamentales et des disciplines essentielles pour les entreprises.

D'autres similitudes, plus concrètes sont identifiables. Métrologie et pédagogie ont un même objectif: la recherche de la conformité par la mesure d'écarts.

Il s'agit également d'activités au service d'autres activités. Une méthodologie rigoureuse permettra d'atteindre l'efficience. Activités transverses dans l'entreprise, l'art de convaincre y est crucial mais difficile.

\section{La recherche de la conformité}

\subsection{Conformité métrologique}

A l'exception des laboratoires d'étalonnage, la finalité de la métrologie dans l'entreprise est de contribuer à apporter la preuve de la conformité du produit aux exigences spécifiées. Pour cela, le métrologue doit déterminer les mesures à entreprendre, mettre en œuvre et maintenir les ressources appropriées, évaluer la validité et la pertinence des résultats de mesure et conserver des informations documentées qui permettent de démontrer la démarche. Pour cela, il doit prévoir la stratégie de mesure la mieux appropriée, la plus fiable et la moins coûteuse. Il doit commencer par identifier le besoin de mesure et les objectifs métrologiques qui vont en découler. La confrontation des résultats de mesure aux objectifs métrologiques permettra l'amélioration du processus de mesure.

\subsection{Conformité pédagogique}

Le pédagogue doit, lui aussi, prévoir une stratégie de formation. Il doit définir les objectifs pédagogiques, énumérer les thématiques qui permettent d'atteindre ces objectifs, estimer le niveau de connaissance des stagiaires afin de planifier le temps nécessaire à l'acquisition de chaque contenu, répertorier la méthode pédagogique appropriée à chaque séquence, prévoir le mode d'évaluation des acquis, utiliser le retour des évaluations pour parfaire la prochaine prestation.

\subsection{Analogie dans la recherche de la conformité}

Bien que le besoin identifié soit totalement différent, l'objectif à atteindre pour un processus de mesure ou pour un processus de formation est la recherche de la conformité. L'analogie ne s'arrête pas là : les moyens à mettre en œuvre regroupent un ensemble de ressources comparables (ressources techniques, humaines, documentaires) et les facteurs de réussite identifiés sont les mêmes. Le livrable est bien différent mais les facteurs de réussite et les éléments d'amélioration sont les mêmes. L'illustration 5 établit la comparaison entre un processus de mesure et un processus de formation et permet d'identifier les principales similitudes.

Illustration 5. Analogie entre un processus de mesure et un processus de formation

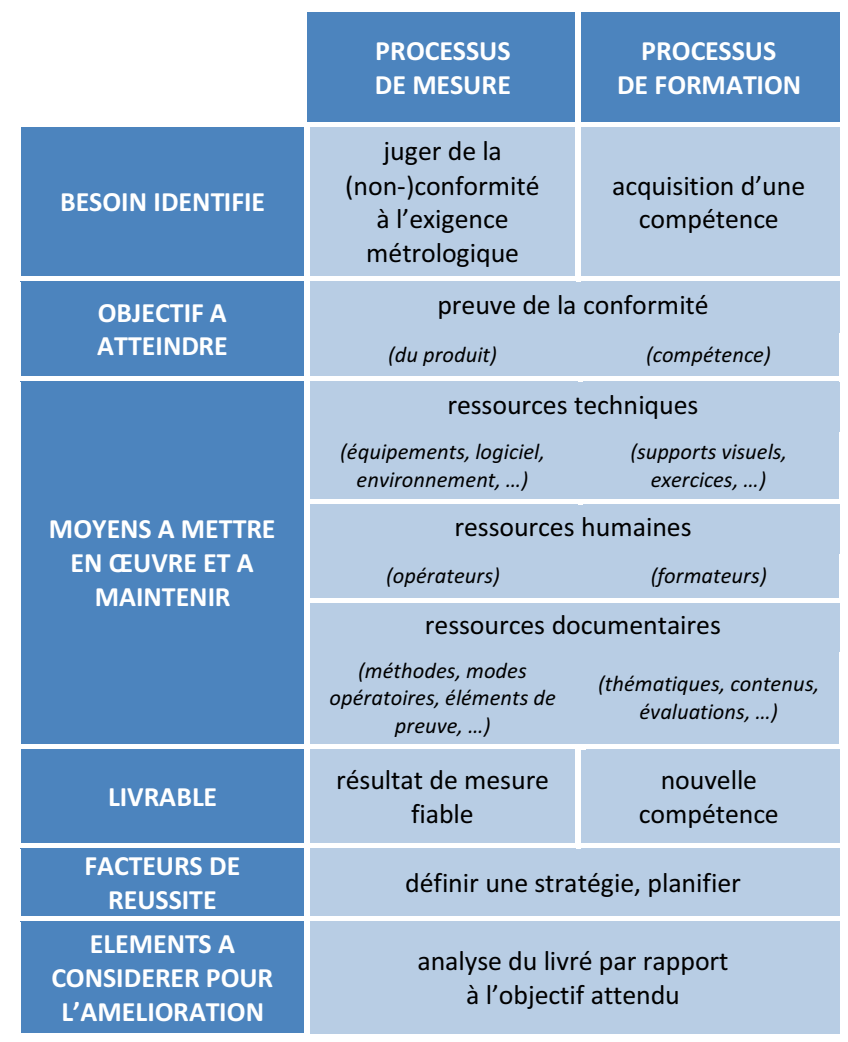

Métrologie et pédagogie visent donc la recherche de la conformité. Pour résumer la démarche, il faut planifier une stratégie à partir d'objectifs définis et analyser les retours pour améliorer.

\section{La mesure des écarts}

\subsection{Les écarts en métrologie}

En métrologie, on mesure des écarts ou des erreurs, la confusion est fréquente entre les deux termes. Les erreurs concernent spécifiquement les équipements de mesure (erreur d'indication, erreur de justesse, ...) et les processus de mesure. La définition du mesurande, si importante en métrologie, prend tout son sens.

Un écart correspond à une différence entre un résultat de mesure et une exigence métrologique. Il est déterminé en confrontant un résultat de mesure à la spécification pour laquelle le processus de mesure a été mis en place. 


\subsection{L'évaluation en formation}

En pédagogie, on mesure l'écart entre le niveau atteint et l'objectif pédagogique fixé, cela consiste à s'assurer que le transfert de connaissances et/ou le développement d'une capacité se fait dans la bonne mesure. C'est-à-dire que l'apprenant s'approprie un savoir, un savoir-faire et/ou un savoir-être selon le pourcentage d'autonomie que s'est fixé le formateur en concevant la prestation. Toutes les évaluations visent à mesurer le niveau d'acquisition d'un apprenant vis-à-vis de sa connaissance initiale et du niveau de l'objectif que le formateur s'est fixé.

\subsection{Analogie des mesures}

Le point commun le plus explicite est sans nul doute le fait de mesurer des écarts. C'est bien entendu le propre de la métrologie mais c'est tout aussi vrai pour la pédagogie.

L'action du métrologue comme du pédagogue se termine par une évaluation. Le résultat obtenu est comparé à l'objectif fixé afin de maintenir ou d'améliorer le niveau de performance nécessaire.

\section{Des activités au service d'autres activités}

Autre point commun, il s'agit d'activités au service d'autres activités. Elles ne sont pas une fin en soi. Leur légitimité vient précisément de la rigueur de leur méthodologie. Outre une organisation rigoureuse, il est également nécessaire de savoir convaincre les partenaires de son utilité. En métrologie comme en pédagogie, il est préférable de savoir constituer son réseau, connaître son environnement pour mieux convaincre, utiliser les techniques de communication.

En effet, le pédagogue n'est pas autonome. Il met la pédagogie au service d'un contenu technique. Il n'existe pas non plus d'entreprise de métrologie. Ce sont des services aux compétences transversales qui œuvrent, au sein d'industries ou de laboratoires, pour d'autres entités. Il est donc préférable de savoir trouver le ton juste et le moment opportun pour collaborer avec les services opérationnels.

\section{Des compétences et des outils communs}

\subsection{Des compétences transverses}

La pratique de la métrologie ou de la pédagogie requière des compétences d'anticipation, d'analyse, de synthèse et de structuration.

En effet, le métrologue et le formateur ont une position stratégique. Le premier est le chef d'orchestre du système de management de la mesure dans son entreprise. A ce titre, il définit, il documente et il s'assure des compétences et des responsabilités des personnes impliquées. Le second, est le facilitateur qui crée l'environnement propice à l'acquisition de compétences.

\subsection{Etre légitime pour obtenir l'adhésion}

Pour être efficace, ils doivent chacun savoir déléguer, faciliter, décider et accompagner. Ils doivent passer de la coordination des tâches à la synchronisation des rôles. Tout comme les managers, ils doivent être capables de gérer l'intelligence d'un groupe. Que la personne manage, gère un projet de métrologie, dispense une formation, elle doit apprendre à passer du stade d'expert qui détient la solution, à personne ressource d'un groupe d'experts sur laquelle les autres peuvent s'appuyer pour évoluer, puis à celui qui crée les conditions pour que les autres puissent évoluer. Cette transformation est nécessaire pour que l'expertise d'une personne puisse se déployer.

Illustration 6. Evolution du métrologue et du formateur [5]

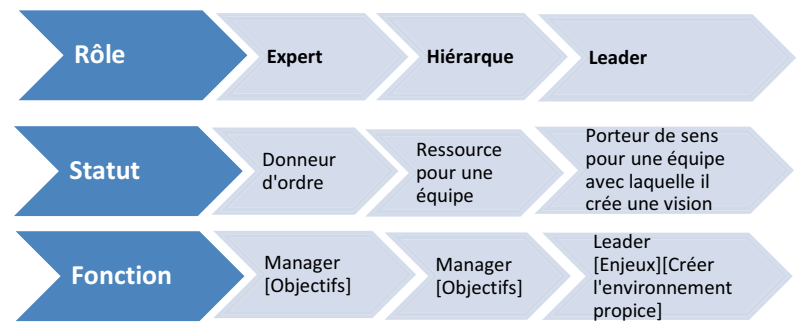

\subsection{L'art de convaincre}

Le métrologue et le formateur ont besoin de convaincre afin de donner sens à leur travail. Convaincre d'autres services pour le métrologue et convaincre les apprenants pour le formateur. Cela implique de maîtriser certains mécanismes de communication. Bien communiquer, c'est d'abord savoir motiver et juguler les conflits.

Illustration 7. Les 2 axes de la communication

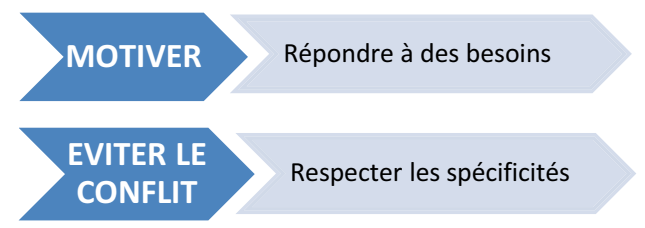

Cela revient à savoir répondre à 3 types de besoins. Le besoin de reconnaissance au sein d'un groupe peut s'illustrer au travers de trois questions : Suis-je suffisamment important pour que le groupe ne m'abandonne pas? Suis-je suffisamment compétent pour ne pas être humilié ? Suis-je suffisamment aimable pour que le groupe ne me rejette pas? [6]. Ces trois questions renvoient aux trois peurs de l'enfance. Le second besoin est celui de la stimulation. C'est tout l'art du formateur de positionner le contenu du stage au juste niveau de difficulté afin de préserver la confiance en soi, la curiosité et l'envie de s'impliquer de l'apprenant. Pour convaincre, le métrologue doit trouver ce même 
équilibre chez ses interlocuteurs pour les inciter à le suivre. Le troisième besoin est celui de structure. Pour s'engager, une personne a besoin d'un cadre qui spécifie : qui fait quoi ? où ? comment? et pourquoi ? Tant qu'une réponse n'est pas apportée à ces trois besoins, il est peu probable que la personne soit convaincue et, sans conviction, il n'y a pas d'engagement.

«Traitez les gens comme s'ils étaient ce qu'ils pourraient être et vous les aiderez à devenir ce qu'ils sont capables d'être », écrivait Goethe [7]

Illustration 8. Les besoins de reconnaissance au sein d'un groupe

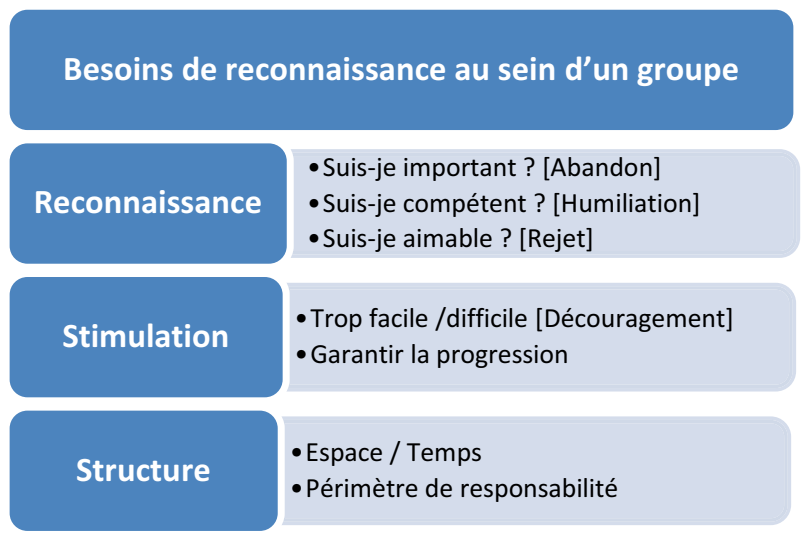

Métrologue et formateur doivent convaincre pour garantir l'engagement des collègues ou des apprenants.

\subsection{La nécessité d'organisation}

Pour l'organisation et la planification, la gestion par projet est utile.

La gestion de projet est un outil pour aider à gagner en légitimité, convaincre sa hiérarchie et faire adhérer les équipes pour porter le changement dans une maîtrise Coûts / Qualité / Délais. Elle peut se définir comme un effort temporaire de nature transverse, borné par un début et une fin, et qui a pour objectif de produire un (des) livrable(s) spécifique(s) répondant à un besoin client préalable défini et validé par le(s) commanditaire(s) du projet. Les points forts de l'organisation par projet sont d'avoir des objectifs et un contenu clairement définis et de s'assurer qu'ils sont partagés. Le travail d'initiation et de planification, d'organisation et de mobilisation des équipes, de gestion des risques est primordial. Pour être efficace, le responsable projet doit savoir déléguer, faciliter, décider et accompagner. Ces définitions pourraient tout autant se reporter à un projet de métrologie ou de formation.

L'installation d'un laboratoire de métrologie, le développement d'un nouveau processus de raccordement interne, l'investissement d'un nouvel étalon ou l'optimisation de la gestion d'un parc d'équipements de mesure sont autant de projets portés par un métrologue. Bien au-delà des connaissances, le succès dans l'atteinte des objectifs de chacun d'eux dépend de la préparation, de la planification, du suivi et de l'accompagnement des équipes mobilisées dans une démarche maîtrisée Coûts / Qualité / Délais.

La garantie du succès d'une formation passe par une préparation rigoureuse et une animation dynamique. La création d'un stage de formation est un projet qui consiste à déterminer des objectifs, rédiger un contenu, sélectionner des méthodes pédagogiques, organiser une chronologie dans un temps imparti, composer puis guider une équipe, créer une grille d'évaluation, assurer une amélioration continue dans la maîtrise Coûts / Qualité / Délais.

\section{La pédagogie de la métrologie}

\subsection{L'addition des difficultés}

Enfin, lorsqu'il s'agit de la pédagogie de la métrologie, les difficultés s'additionnent et le choix des méthodes et outils d'apprentissage sont stratégiques.

\subsection{L'évolution de la pédagogie}

Dans l'entreprise, rappelons que la formation professionnelle continue doit permettre l'acquisition de compétences identifiables et mesurables et déterminer des objectifs en termes de connaissances, de compétences ou de qualification à acquérir.

Illustration 9. Evolution de la pédagogie en entreprise

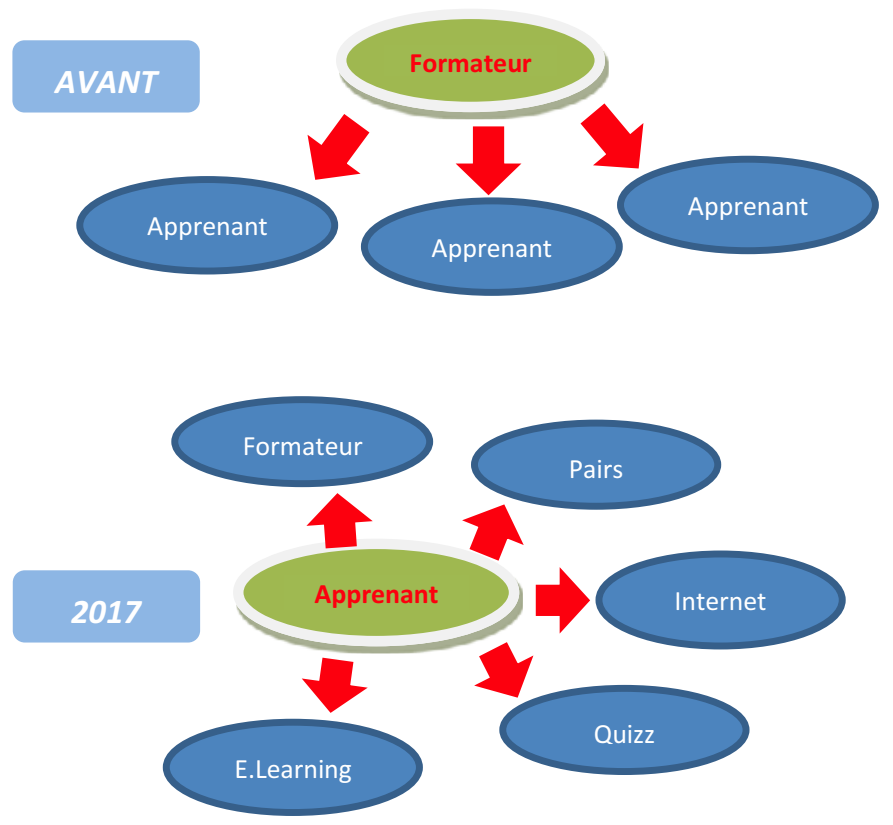

La formation est centrée sur le stagiaire et non plus sur l'intervenant comme le montre l'illustration 9. Elle s'est fortement adaptée aux nouveaux enjeux du management et se fixe pour objectifs de formation de :

- développer des capacités d'adaptation pour franchir des obstacles;

- utiliser des compétences pour appliquer et rebondir ; 
- développer la créativité ;

- construire une réflexion personnelle en se nourrissant du collectif.

Les méthodes pédagogiques ont été adaptées en conséquence :

- création d'un référentiel de compétences spécifiques à une fonction ;

- départ d'un problème auquel on apporte une solution ;

- le stagiaire agit plus qu'il n'écoute ;

- un stage de formation est un écosystème.

La trame pédagogique est construite en fonction de challenges à résoudre qui correspondent à des compétences métier. Il s'agit d'orienter la formation vers des résultats opérationnels.

Illustration 10. Exemple de trame pédagogique

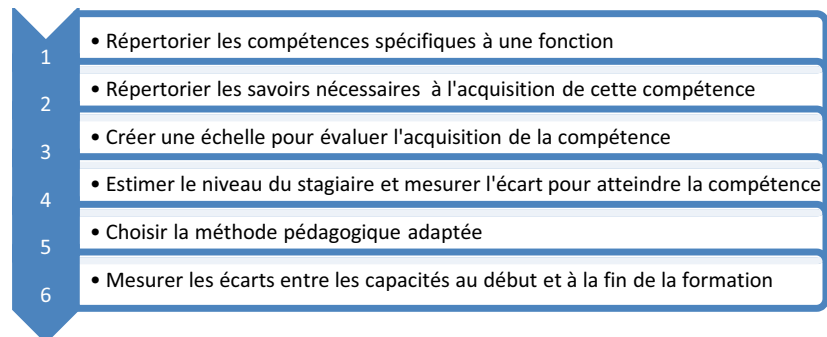

Former implique de bien connaître les enjeux d'une fonction métier à laquelle le stage s'adresse.

\subsection{La pédagogie de la métrologie au LNE}

Depuis 1981, le LNE dispose d'un centre de formation qui propose des prestations de formations en métrologie, dispensées par le personnel métrologue formé aux techniques pédagogiques. Très tôt, le laboratoire a pris conscience du lien étroit qui existe entre métrologie et pédagogie et a souhaité en faire bénéficier ses clients. La pédagogie y évolue car les techniques d'apprentissage doivent s'adapter aux exigences du marché.

Le centre de formation du LNE a été créé pour répondre à la demande des clients d'être accompagnés sur des sujets réglementaires et des expertises. Au tout début, les experts du LNE témoignaient de leur activité et proposaient des stages permettant de développer des capacités techniques. Puis, ils se sont formés à l'andragogie et aux techniques de communication orale dans un premier temps et aux approches ludiques ensuite. Aujourd'hui, ils arborent de plus en plus la posture de facilitateur pour répondre aux attentes des clients; diversifient les méthodes pédagogiques et proposent de nombreuses simulations au plus près de la réalité terrain des stagiaires, proposent des visites de laboratoires et des auto-évaluations. La gamme Métrologie est composée d'un parcours de formation à partir de la thématique «comment assurer la performance des résultats de mesure, d'essais et d'analyse » et se décline en compétences nécessaires à la fonction. Les stages proposés abordent les problématiques résumées dans l'illustration 11 .

Illustration 11. Parcours de formation métrologie proposé par le LNE

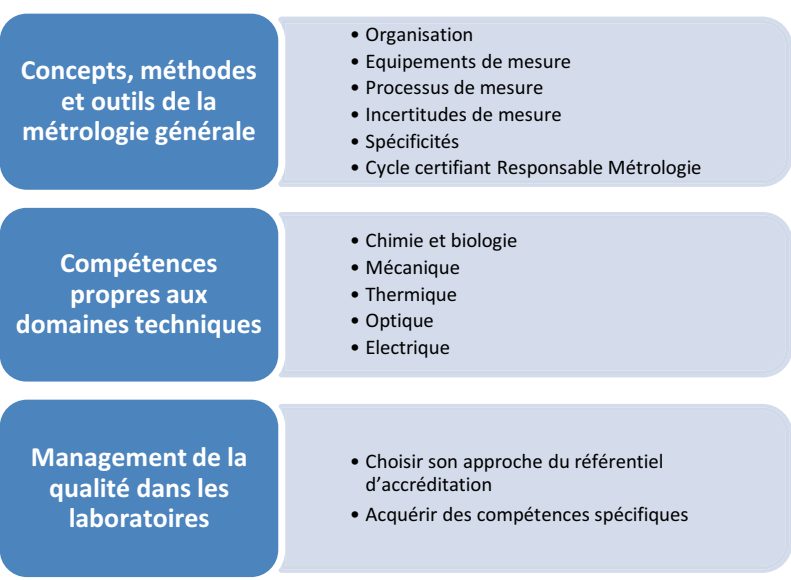

\subsection{L'importance des outils pédagogiques}

\subsubsection{L'alternance}

Différents outils pédagogiques utilisés en alternance permettent d'augmenter la rétention du stagiaire. L'illustration 12 représente l'évolution du taux de rétention en fonction de la méthode utilisée

Illustration 12. Taux de rétention du contenu après 24 heures selon la méthode d'enseignement [8]

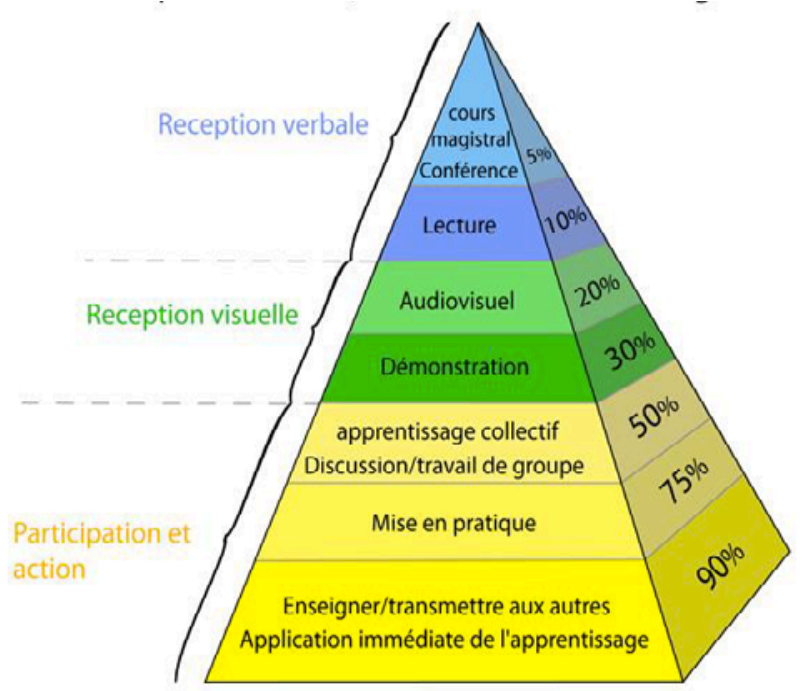

«Tu me dis, j'oublie. Tu m'enseignes, je me souviens. Tu m'impliques, j'apprends.», cette citation de Benjamin Franklin résume la nécessité d'impliquer l'apprenant dans la formation.

L'illustration 13 liste les principaux outils pédagogiques en y précisant leur utilité. 
Illustration 13. Principaux outils pédagogiques

\begin{tabular}{|c|l|}
\hline Outil pédagogique & Utilité \\
\hline Exposé, cours magistral & $\begin{array}{l}\text { - Transférer un contenu non maîtrisé } \\
\text { par l'apprenant de façon exhaustive } \\
\text { - Fournir des éléments nécessaires à la } \\
\text { compréhension de la formation }\end{array}$ \\
\hline Lecture & $\begin{array}{l}\text { - Découvrir un contenu non maîtrisé par } \\
\text { I'apprenant }\end{array}$ \\
\hline - Solliciter l'ouïe pour mémoriser et \\
commencer à structurer sa pensée \\
- Engager l'apprenant \\
\hline Démonstration & $\begin{array}{l}\text { - Découvrir un contenu non maîtrisé par } \\
\text { I'apprenant }\end{array}$ \\
\hline - Solliciter la vue pour mémoriser et \\
commencer à structurer sa pensée
\end{tabular}

\subsubsection{Les activités ludiques}

Les activités ludiques se sont fortement développées. Se former permet de faire un bilan de son activité et de ses compétences et les compétences transverses se développent plus aisément par le jeu. La formation s'appuie sur l'expérience personnelle des participants et les jeux de mise en situation permettent à un groupe de vivre une situation qui simule la réalité de l'entreprise.

En outre, les jeux aident à développer la créativité des participants. La créativité développée lors d'une formation peut prendre différents aspects :

- Imaginer différentes solutions originales pour résoudre un problème ;

- Etre capable d'apercevoir des relations entre les faits jamais rapprochés jusque-là ;

- Etre flexible et sortir de son domaine pour trouver des voies d'amélioration;

- Pratiquer le tâtonnement expérimental;

- Avoir le droit à l'erreur ;

- Enchaîner ses propositions sur celles des autres ;

- Résoudre des problèmes, chercher des solutions, se concerter, donner des réponses, imaginer des façons de faire, donner son avis, argumenter une opinion.

L'illustration 14 propose une liste non exhaustive des principaux jeux utilisés en formation en faisant le lien avec la compétence transverse travaillée.
Illustration 14. Principaux jeux utilisés en formation

\begin{tabular}{|c|c|c|}
\hline Jeu & Consigne & $\begin{array}{l}\text { Compétences } \\
\text { transverses travaillées }\end{array}$ \\
\hline 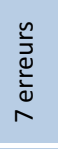 & $\begin{array}{l}\text { Retrouver les erreurs dans } \\
\text { un document }\end{array}$ & $\begin{array}{l}\text { - Distinguer le vrai du faux } \\
\text { - Repérer rapidement une } \\
\text { erreur } \\
\text { - Corriger une erreur }\end{array}$ \\
\hline 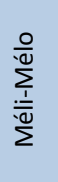 & $\begin{array}{l}\text { Retrouver les bonnes } \\
\text { correspondances entre deux } \\
\text { éléments }\end{array}$ & $\begin{array}{l}\text { - Savoir mettre en lien des } \\
\text { éléments } \\
\text { - Distinguer le vrai du faux } \\
\text { - Suivre une chronologie, un } \\
\text { processus }\end{array}$ \\
\hline 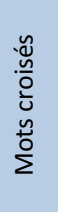 & $\begin{array}{l}\text { Positionner les mots sur une } \\
\text { grille en fonction des } \\
\text { définitions données }\end{array}$ & $\begin{array}{l}\text { - S'approprier le jargon } \\
\text { - Etre précis dans sa com- } \\
\text { munication } \\
\text { - Savoir ce qu'implique un } \\
\text { mot en termes d'actions à } \\
\text { mener }\end{array}$ \\
\hline 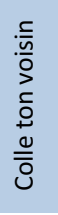 & $\begin{array}{l}\text { Chaque participant pose une } \\
\text { question sur le contenu de la } \\
\text { formation à son voisin de } \\
\text { gauche (il connaît la répon- } \\
\text { se) et valide la qualité de la } \\
\text { réponse donnée }\end{array}$ & $\begin{array}{l}\text { - Vérifier ce qui a été retenu } \\
\text { et compléter ou corriger si } \\
\text { nécessaire } \\
\text { - Formuler avec précision } \\
\text { - Savoir déléguer, trans- } \\
\text { mettre, convaincre }\end{array}$ \\
\hline 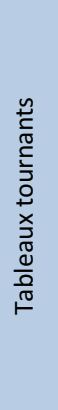 & $\begin{array}{l}\text { Dans un premier temps, } \\
\text { deux groupes travaillent } \\
\text { chacun sur une partie d'une } \\
\text { thématique et proposent } \\
\text { leurs réponses sur un ta- } \\
\text { bleau; dans un second } \\
\text { temps, les groupes changent } \\
\text { de tableau et complètent ou } \\
\text { corrigent le travail de leurs } \\
\text { collègues; dans un troisième } \\
\text { temps, l'évaluateur fait la } \\
\text { synthèse et corrige si besoin }\end{array}$ & $\begin{array}{l}\text { - Harmoniser les représen- } \\
\text { tations } \\
\text { - Corriger les idées fausses } \\
\text { - Savoir définir un terme } \\
\text { - Savoir argumenter, con- } \\
\text { vaincre, synthétiser }\end{array}$ \\
\hline
\end{tabular}

\section{Synthèses et perspectives}

La métrologie est omniprésente dans les entreprises. De plus en plus de techniciens et de référents qualité ajoutent la charge de Responsable Métrologie à leur fonction. Ils ont besoin d'avoir une vue d'ensemble du périmètre de leurs responsabilités. En 2011, un rapport d'étude sur l'offre et les besoins de formation en métrologie soulignait l'insuffisance de l'offre de formation en métrologie en France [9]. Nous avons noté la méconnaisse de la métrologie et le manque de vue synoptique des nouveaux responsables métrologie.

Sans s'en rendre forcément compte, le manque de connaissances métrologiques dans les entreprises n'a pas entraîné de conséquences négatives généralisées (le surdimensionnement y est pour quelque chose...). Ce trouble, lui généralisé, trouve son explication dans la certitude que la métrologie concerne exclusivement les instruments de mesure. L'organisation très variée de la fonction métrologie dans les entreprises témoigne aussi des différences de vue sur l'importance de celle-ci (toutes les entreprises n'ont pas nommé un Responsable Métrologie par exemple). Si mal connue, si mal maîtrisée... 


\section{Références}

En renforçant la formation, le métrologue va pouvoir devenir le garant de la fiabilité des résultats de mesure, le responsable métrologie le chef d'orchestre du système de management de la mesure. Les compétences techniques seront complétées par des compétences transverses.

L'offre de formation est nécessaire si tant est qu'elle propose des stages qui permettent de s'approprier, à la fois la vision globale et la rigueur de la métrologie. Les approches managériales en mode projet, la posture du chef de projet et du formateur comme facilitateur, l'introduction de l'approche ludique qui met l'apprenant au centre du processus de formation sont autant d'évolutions qui garantissent un bel avenir à la formation en métrologie.

Les décisions industrielles sont fondées sur des résultats de mesures fiables et partagés, cette finalité oubliée va apparaître aux entreprises. L'utilisation des nouvelles approches pédagogiques peut aider la métrologie à (re)trouver ses lettres de noblesse.
1. Marie-Ange COTTERET, Rapport de thèse " Métrologie et enseignement » (2003)

2. Larousse [consulté le 06/06/2017]

3. Sophie COURAU, «Les outils d'excellence $d u$ formateur ", (ESF 2001)

4. Disponible à l'adresse : http://andragogiesformations.over-blog.com/pages/ L_ANDRAGOGIE_cest_quoi-6503995.html

5. Vincent LENHARDT, "Les responsables porteurs de sens » (1982)

6. Will SCHUTZ, "L'élément humain » (1994)

7. Johann Wolfgang GOETHE, écrivain, homme politique et savant allemand [1749-1832]

8. Disponible à l'adresse : http://www.pearltrees.com/ christophecoussit/concepts-pedagogiquesbase/id8774877/item127782725

9. Ecole des mines de DOUAI, Rapport final d'étude sur l'offre et les besoins de formation en métrologie, enquête réalisée pour le compte du Ministère de l'économie, des finances et de l'industrie (2011) 